\title{
Efficacy of a New Protocol of Premixed 70/30 Human Insulin in Haitian Youth with Diabetes
}

\author{
Eddy Jean-Baptiste (D) Philippe Larco (D) · Julia von Oettingen (D) \\ Graham David Ogle (D) - Keddy Moïse · Evelyne Fleury-Milfort • \\ Rodolphe Paul · René Charles · Nancy Charles Larco
}

Received: June 9, 2021 / Accepted: July 23, 2021 / Published online: August 11, 2021

(C) The Author(s) 2021

\begin{abstract}
Introduction: Controlling insulin-treated diabetes is challenging in low-resource settings where only Neutral Protamine Hagedorn (NPH), regular $(\mathrm{R})$ and premixed insulin formulations are available, self-monitoring of blood glucose (SMBG) supplies are scarce and food insecurity is common. We examined the impact of a treatment protocol that includes sliding scalebased 70/30 insulin adjustments in Haiti.
\end{abstract}

Methods: Thirty young patients aged 11-28 years with diabetes treated with premixed 70/30 insulin twice daily were included

E. Jean-Baptiste $(\square) \cdot$ P. Larco - K. Moïse .

E. Fleury-Milfort · R. Paul · R. Charles · N. C. Larco

Haitian Foundation for Diabetes and Cardiovascular

Diseases (FHADIMAC), 208, Lalue, HT 6114 Port-au-

Prince, Haiti

e-mail: eddyjeanba@yahoo.com

P. Larco

e-mail: philippel@hotmail.com

J. von Oettingen

Research Institute of the McGill University Health

Centre, 1650 Cedar Avenue, Montreal, QC H3G

1A3, Canada

J. von Oettingen

Montreal Children's Hospital, 1001 Boulevard

Decarie, Montreal, QC H4A 3J1, Canada

G. D. Ogle

Life for a Child Program, Diabetes NSW, 26 Arundel

St., Glebe, NSW 2037, Australia in the study. The participants performed one or two daily self-monitoring of blood glucose (SMBG) tests and attended our diabetes clinic monthly. They were randomized to two treatment groups, with one group remaining on the 70/30 insulin formulation (group 70 [G70]) and the other group switching to self-mixed $\mathrm{NPH}+\mathrm{R}$ (group NR [GNR]). Sliding scales for insulin correction doses and meal insulin doses were designed based on the total daily insulin dose (TDD), carbohydrate ratio and insulin sensitivity factor. SMBG tests and insulin were administered before the morning and evening meals. The frequency of visits to the diabetes clinic was increased to biweekly during a 14-week follow-up.

Results: Fifteen patients of each group were included in the analysis. Baseline characteristics, increase in total daily dose and number of missed SMBG tests and skipped meals at 14 weeks did not differ between the two groups. Hemoglobin A1c (HbA1c) decreased from 9.5\% (interquartile range [IQR] 8.8, 10.5) $(80.3 \mathrm{mmol} / \mathrm{mol})$ to $8.0 \%$ (IQR $7.1 \%, 9.0 \%$ ) $(63.9 \mathrm{mmol} / \mathrm{mol})$ in $\mathrm{G} 70(p=0.01)$, and from $10.6 \%$ (IQR $8.1, \% 13.1) \%(92.4 \mathrm{mmol} / \mathrm{mol})$ to $9.0 \%$ (IQR $7.6 \%, 9.6 \%)(74.9 \mathrm{mmol} / \mathrm{mol})$ in GNR $(p=0.10)$, with no significant betweengroup difference in reductions $(p=0.12)$. No serious acute complications were reported. Stopping the use of sliding scales and resuming monthly visits increased HbA1c to values not 
significantly different from baseline in both groups after 15 weeks.

Conclusion: The use of sliding scales adjusted for missed SMBG tests and skipped meals, and frequent clinic visits that focus on patient selfmanagement education significantly improved glycemic control in the patients with youthonset diabetes in our study treated with premixed 70/30 human insulin in a low-resource setting.

Keywords: Insulin-treated diabetes; Lowresource setting; Missed self-monitoring of blood glucose tests; Premixed insulin; Selfmanagement education; Self-mixed insulin; Skipped meals; Sliding scales; Youth-onset diabetes

\section{Key Summary Points}

Controlling diabetes in young patients (youth-onset diabetes) who are treated with insulin is challenging in lowresource settings where the treatment relies only on Neutral Protamine Hagedorn (NPH), regular inusulin and premixed biphasic human insulin formulations.

We hypothesized that metabolic control could be improved in these patients by providing better self-management education in combination with the use of sliding scales which provide for patientadapted self-adjustment of premixed $70 / 30$ insulin doses that account for missed self-monitoring of blood glucose (SMBG) tests and skipped meals.

The results showed that glycemic control significantly improved with this treatment protocol.

These data call for new strategies for better metabolic control in patients living in limited-resource settings where cuttingedge therapies are still lacking.

\section{INTRODUCTION}

Glycemic control is the single most important predictor of an increased risk for diabetes-related complications [1]. Analog basal-bolus insulin regimens are the standard of care in high-income settings where increasingly smarter insulin delivery systems are being developed and used to best mimic physiologic insulin needs. Even with such cutting-edge therapies, achieving glycemic targets [2] in young patients with diabetes (youth-onset diabetes) is very challenging [3] due to multiple factors, including lifestyle, developmental, psychological and hormonal changes.

Patients of ethnic minorities and lower socioeconomic status are disproportionately at risk of poor glycemic control [3-5]. This is especially true in less-resourced settings, such as in low-income countries where analog insulins and insulin pumps are mostly inaccessible or unaffordable [6]. Instead, patients and healthcare providers must rely on Neutral Protamine Hagedorn (NPH), regular (R) and premixed biphasic human insulin formulations [6]. The pharmacodynamics of these insulins call for regular meals and snacks to "feed the insulin" when it peaks in order to avoid hypo- or hyperglycemia. In a context where only minimal care standards [6] are available, glucometers and glucose test strips are scarce and food insecurity is common, it is exceedingly difficult to achieve glycemic control among insulintreated patients with youth-onset diabetes [6-8]. In Haiti, youth-onset diabetes is mainly treated with twice-daily human insulin injection regimens; for the most part these include premixed insulin consisting of 70\% NPH and $30 \% \mathrm{R}$ (70/30 insulin), with NPH and R insulins given as separate injections to a lesser extent. The reason for the higher use of premixed insulin among these young patients is essentially due to their low education level, which may be a limiting factor in the self-management of insulin mixing, and the greater availability of premixed insulin on the market compared with NPH insulin.

The few publications on Haitian youth with diabetes treated with insulin have shown poor 
glycemic control, with an average hemoglobin A1c (HbA1c) of $>11 \%[7,8]$. Given the economic challenge to obtain analog insulins and increase the number of daily self-monitoring of blood glucose (SMBG) tests, as well as the inability to eradicate food insecurity and irregular access to daily meals and snacks, it became necessary to seek a strategy by which to improve glycemic control despite these context-specific barriers. Therefore, we hypothesized that patient-adapted self-adjustment of premixed insulin doses that accounts for missed SMBG tests and skipped meals could improve glycemic control in youth with diabetes. The aim of this study was to determine the impact of a sliding scale-based insulin adjustment protocol that would account for missed SMBG tests and skipped meals, in young patients with diabetes. The primary objective was to improve HbA1c after the adoption of the new protocol. The secondary objective was to compare the outcomes of a group of patients treated with 70/30 insulin with those of another group treated with self-mixed $\mathrm{NPH}+\mathrm{R}$ insulins under the same follow-up conditions.

\section{METHODS}

\section{Study Design}

This was a prospective open-label randomized controlled trial of patients with insulin-treated youth-onset diabetes in Port-au-Prince, Haiti, from 21 October 2017 to 17 May 2018.

\section{Sample Size}

The sample size calculation was based on the primary outcome reduction in HbA1c in G70. Thirty-two patients (16 in each group) were required to detect a 1 percentage point reduction in $\mathrm{HbA} 1 \mathrm{c}$ with $80 \%$ power and a $5 \%$ level of significance.

\section{Participants}

We recruited young patients from the FHADIMAC (Haitian Foundation for Diabetes and
Cardiovascular Diseases) outpatient clinic, which is the largest pediatric diabetes outpatient setting in Haiti, with a catchment area that includes the capital city of Port-au-Prince and the surrounding cities and suburbs. Inclusion criteria were age 11-28 years and treatment with 70/30 human insulin twice daily. Exclusion criteria were renal failure, severe visual impairment and pregnancy. Written informed consent was obtained from adult patients and from adults legally responsible for minors. The study protocol was reviewed and approved by the Haitian National Bioethics Committee of Haitian Ministry of Health (Ref: 1617-55). It was performed in accordance with the Helsinki Declaration of 1964, and its later amendments.

\section{Baseline and Study Treatment Protocols}

The baseline treatment protocol included twicedaily insulin doses, one or two SMBG tests per day, a monthly 10-minute visit and the recommendation to present the SMBG and insulin adjustment reports at each visit. The carbohydrate $(\mathrm{CHO})$ counting method, which included Haitian meals, was refreshed.

Patients were provided with free SMBG supplies, insulin and syringes through the Life for a Child Program [9]. Hyperglycemic crises including diabetic ketoacidosis (DKA) and hyperglycemic hyperosmolar state (HHS) were defined according to the consensus guidelines of the International Society for Pediatric and Adolescent Diabetes (ISPAD) [10]. The biochemical criteria, which are generally associated with a variable clinical presentation, for the diagnosis of DKA were: blood glucose $(\mathrm{BG})>$ $200 \mathrm{mg} / \mathrm{dL}(11 \mathrm{mmol} / \mathrm{L})$, venous $\mathrm{pH}<7.3$ or bicarbonate $<15 \mathrm{mmol} / \mathrm{L}$, ketonemia or ketonuria. The criteria for HHS included: BG $>600$ $\mathrm{mg} / \mathrm{dL}(33.3 \mathrm{mmol} / \mathrm{L})$, venous $\mathrm{pH}>7.25$ or arterial $\mathrm{pH}>7.30$, serum bicarbonate $>15$ $\mathrm{mmol} / \mathrm{L}$, small ketonuria, absent to mild ketonemia, effective serum osmolality $>320$ $\mathrm{mOsm} / \mathrm{kg}$, altered consciousness (e.g. obtundation, combativeness) or seizures. Severe hypoglycemia was defined in adulthood as an asymptomatic hypoglycemic event associated with severe cognitive impairment (including 
coma and convulsions) requiring external assistance by another person to actively take corrective actions. In childhood, it was defined as an event associated with severe neuroglycopenia resulting in coma or seizure and requiring parenteral therapy (glucagon or intravenous glucose) [11].

From the list of all patients who regularly attended the clinic during the 12 weeks prior to study start, we selected those who met the inclusion criteria. After removing the selected subjects who presented one or more exclusion criteria, 32 remained as study participants. They were assigned via fixed block randomization (block size 4) to remain on $70 / 30$ (group 70 [G70], $n=16$ ) or to switch to self-mixed NPH + R (group NR [GNR], $n=16$ ), with twice-daily injections. In GNR, NPH insulin accounted for $50 \%$ of the total daily dose (TDD). Both groups were instructed to consume about $60 \mathrm{~g}$ of $\mathrm{CHO}$ at each of the three daily meals and up to two 15-g snacks per day at mid-morning and midafternoon, respectively. A carbohydrate-to-insulin ratio was calculated for each patient using the 450 rule ( 450 divided by TDD) to obtain an estimate of how many grams of $\mathrm{CHO}$ can be consumed for each 1 unit of insulin given [12]. Meal insulin doses (MID), representing the insulin $\mathrm{R}$ doses required for the $\mathrm{CHO}$ content in a meal, were calculated for $60 \mathrm{~g}$ and other amounts of $\mathrm{CHO}$. An insulin sensitivity factor (that refers to the $\mathrm{mg} / \mathrm{dL}$ drop in BG caused by 1 unit of rapid acting or regular insulin taken in a fasting or pre-meal state) was calculated by dividing the constant 1700 by the TDD [12-14]. Planned dose is defined as the previous dose adjusted, when needed, to its effect on BG.

A sliding scale was provided to all patients according to their randomization and TDD. For patients in G70, the front side displayed insulin correction doses (ICD) for different BG ranges (see Table 1) and were used to adjust or determine the 70/30 insulin doses shown in Table 2 when applicable (meal skipped and BG measure missed, or mid-day BG, when performed, $>240$ $\mathrm{mg} / \mathrm{dL}$ ). The other cases were based on a retrospective algorithm (planned dose). For patients in GNR, the front side of the sliding scale displayed the ICD (Table 1) and the pre-meal insulin $\mathrm{R}$ doses (ICD + MID) related to $60 \mathrm{~g}$ of
$\mathrm{CHO}$ for different BG ranges. However, adjustment of the twice-daily doses of NPH insulin was based on a retrospective algorithm (planned dose). Additional instructions were provided on pre-meal insulin $\mathrm{R}$ dose adjustment for estimated CHO loads other than $60 \mathrm{~g}$. The corresponding part (G70 or GNR) of Table 2 was positioned on the back of the sliding scales to determine insulin doses based on the presence of pre-meal BG tests and meals.

SMBG and insulin were routinely administered before morning and evening meals. The pre-meal glycemic target was $80-130 \mathrm{mg} / \mathrm{dL}$. Any BG test not done in the morning or evening with or without a meal was considered missed. Any meal not taken among the three standard daily meals was considered skipped. Patients were encouraged to perform SMBG at the time of any skipped meal, before the next meal following $\mathrm{BG}<80 \mathrm{mg} / \mathrm{dL}$ or if needed (symptoms of hypo- or hyperglycemia, or unexplained malaise).

All patients visited the diabetes clinic twice a month for a 14-week intervention period. The visit duration was also liberalized to allow for better communication on SMBG, insulin adjustment rules and meal $\mathrm{CHO}$ load. All visits were timed to track duration. At the end of this 14-week period and up to 29 weeks post-enrollment, while the current insulin types in each group were maintained, the use of sliding scales was discontinued and the previous 10-min-long, once-monthly visit was resumed.

\section{Data Collection}

Clinical and demographic information was collected from the patients' records. At 14 weeks, patients were re-evaluated in terms of glycemic outcomes, insulin needs, number of meals taken and number of BG tests performed. HbA1c was performed with a point-of-care DCA Vantage machine (Siemens Healthineers AG, Erlanden, Germany) on study day 1 (day of training and provision of all necessary supplies) and at 14 and 29 weeks. 
Table 1 Insulin correction doses

\begin{tabular}{lll}
\hline Pre-meal blood glucose $(\mathbf{m g} / \mathbf{d L})$ & $\begin{array}{l}\text { Total daily insulin dose }<\mathbf{2 0} \mathbf{U} \\
\mathbf{R} \text { insulin dose }\end{array}$ & $\begin{array}{l}\text { Total daily insulin dose } \mathbf{2} \mathbf{2 0} \mathbf{~} \\
\mathbf{R} \text { insulin dose }\end{array}$ \\
\hline$<70$ & No ICD and treat hypoglycemia & No ICD and treat hypoglycemia \\
$70-79$ & No ICD & No ICD \\
$80-150$ & No ICD & No ICD \\
$151-180$ & $0.5 \mathrm{U}$ & $1 \mathrm{U}$ \\
$181-210$ & $0.5 \mathrm{U}$ & $2 \mathrm{U}$ \\
$211-240$ & $0.5 \mathrm{U}$ & $3 \mathrm{U}$ \\
$241-270$ & $1.5 \mathrm{U}$ & $4 \mathrm{U}$ \\
$271-300$ & $1.5 \mathrm{U}$ & $5 \mathrm{U}$ \\
$>300$ & $2 \mathrm{U}$ & $6 \mathrm{U}$ \\
\hline
\end{tabular}

$I C D$ Insulin correct dose, $R$ regular

\section{Statistical Analysis}

The Epi Info version 7.1.3.0 software package was used for data entry and descriptive statistics, and SPSS Statistics version 17.0 (SPSS IBM Corp., Armonk, NY, USA) was used for nonparametric tests and multivariate analysis. We used the Mann-Whitney $U$ test to compare the median between the groups and the chi-squared test for categorical variables. A $p$ value of $<0.05$ was considered to be significant. All analyses were intention-to-treat.

\section{RESULTS}

The complete analysis was performed on 30 participants because two participants left the study during the intervention period for personal reasons. All patients could be assessed as belonging to the lower social class of the general population. The baseline and 14-week characteristics are shown in Table 3. At baseline, the median age at evaluation and diagnosis, rate of patients diagnosed after age 14 years, proportion of females, body mass index (BMI) $Z$-score, duration of diabetes, HbA1c, history of DKA at any stage and TDD of insulin did not differ between the two groups. G70 has a median HbA1c value of $9.5 \%$ (interquartile range [IQR]
$8.8 \%, 11.1 \%)(80.3 \mathrm{mmol} / \mathrm{mol})$ and GNR has a median HbA1c value of $10.6 \%$ (IQR $8.1 \%$, $14.4 \%)(92.4 \mathrm{mmol} / \mathrm{mol})$; the difference was not statistically significant $(p=0.59)$. A wide dispersion of HbA1c values was noted in GNR, with six patients with $\mathrm{HbA} 1 \mathrm{c}>12 \%$ versus one patient in G70. The HbA1c target of $<8 \%(63.9$ $\mathrm{mmol} / \mathrm{mol}$ ) was reached in two patients in each group (13.3\%). None of the patients were obese. None of the patients presented retinopathy nor microalbuminuria. Cataract was found in two patients (one in each group), and one patient in G70 presented suspected glaucoma.

At 14 weeks, the duration of the clinic visit had increased by 7 min compared to the baseline average visiting time of $10 \mathrm{~min}$. Five patients in GNR had persistent difficulty in fully mastering the insulin mixing technique. The HbA1c in G70 patients decreased by $1.5 \%$, reaching a median of $8.0 \%$ (IQR $7.1 \%, 9.2 \%$ ) $(63.9 \mathrm{mmol} / \mathrm{mol})(p<0.01)$; in comparison, the HbA1c in GNR patients decreased by $1.6 \%$, reaching a median of $9.0 \%$ (IQR 7.6\%, 9.9\%) $(74.9 \mathrm{mmol} / \mathrm{mol})(p=0.10)$ (Fig. 1). There was no statistically significant between-group difference in HbA1c reduction $(p=0.12)$. The proportion of patients with HbA1c $<8 \%(63.9$ $\mathrm{mmol} / \mathrm{mol})$ reached $46.7 \%(7 / 15)$ in $\mathrm{G70}(p=$ $0.04)$ and $33.3 \%(5 / 15)$ in GNR $(p=0.39)$; this between-group difference was not statistically 
Table 2 Insulin dose adjustment according to presence of pre-meal blood glucose tests and meals

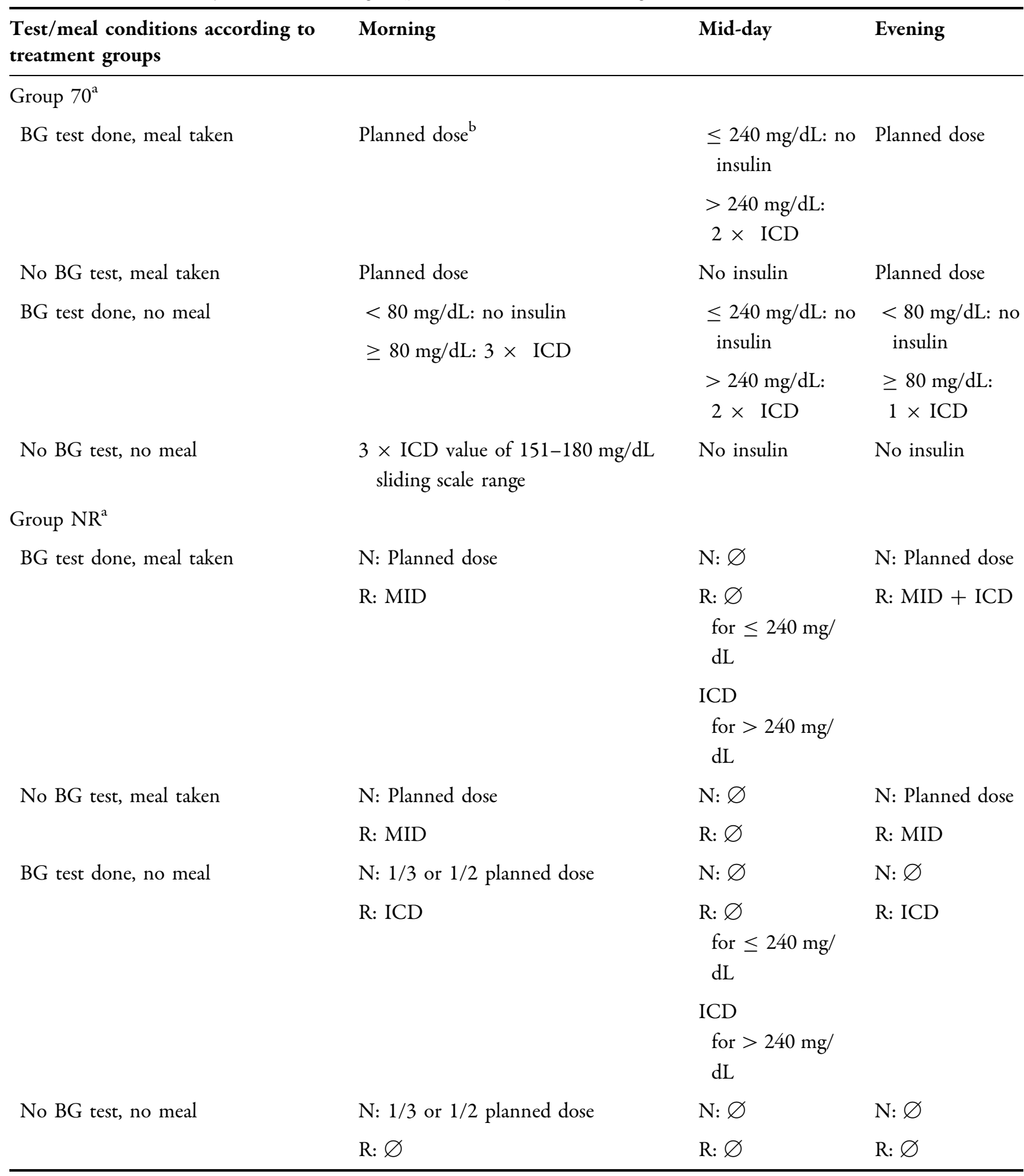

$B G$ Blood glucose, $M I D$ meal insulin dose

${ }^{a}$ Group 70 (G70) were treated with the 70/30 insulin formulation; Group NR (GNR) switched to self-mixed Neutral Protamine Hagedorn $(\mathrm{NPH})+$ regular $(\mathrm{R})$ insulin

b Planned dose is defined as the previous dose adjusted, when needed, to have its effect on BG $\varnothing$ : no insulin 
Table 3 Characteristics of participants at baseline and 14 weeks

\begin{tabular}{|c|c|c|c|}
\hline Patient characteristics & Group $70(n=15)$ & Group N R $(n=15)$ & $p$ value \\
\hline \multicolumn{4}{|l|}{ Characteristics at baseline } \\
\hline Current age, years & $20.0[18.0,23.0]$ & $19.0[15.5,23.5]$ & 0.88 \\
\hline Age at diagnosis, years & $16.0[10.0,19.5]$ & $14.0[11.5,17.5]$ & 0.85 \\
\hline Patients diagnosed after age 14 years & $9(60.0)$ & $7(46.7)$ & 0.71 \\
\hline Female & $9(60.0)$ & $7(46.7)$ & 0.71 \\
\hline BMI $Z$-score & $-0.5[-1.1,0.3]$ & $-0.2[-1.6,0.7]$ & 0.87 \\
\hline Normal BMI $Z$-score ${ }^{a}$ & $12(80.0)$ & $9(60.0)$ & 0.42 \\
\hline Diabetes duration, years & $5.0[2.0,8.5]$ & $4.0[2.5,8.0]$ & 0.98 \\
\hline HbAlc, \% & $9.5[8.8,11.1]$ & $10.6[8.1,14.4]$ & 0.59 \\
\hline HbAlc, \% (mean \pm SD) & $9.8 \pm 2.1$ & $10.8 \pm 2.9$ & 0.27 \\
\hline Patients with $\mathrm{HbAlc}<8 \%$ & $2(13.3)$ & $2(13.3)$ & 1.00 \\
\hline History of diabetic ketoacidosis at any stage & $3(20.0)$ & $3(20.0)$ & 1.00 \\
\hline Total daily insulin dose, $\mathrm{U}$ & $40.0[32.0,55.0]$ & $38.0[26.5,53.0]$ & 0.66 \\
\hline Total daily insulin dose $/ \mathrm{kg}, \mathrm{U}$ & $0.8[0.6$ to 1.0$]$ & $0.8[0.6$ to 0.9$]$ & 0.40 \\
\hline Education & & & 0.39 \\
\hline Elementary & $3(20.0)$ & $5(33.3)$ & \\
\hline Middle School & $2(13.3)$ & $3(20.0)$ & \\
\hline High School & $8(53.3)$ & $4(28.7)$ & \\
\hline Professional & $0(0.0)$ & $1(6.7)$ & \\
\hline University & $1(6.7)$ & $2(13.3)$ & \\
\hline \multicolumn{4}{|l|}{ Characteristics at 14 weeks } \\
\hline Increase in visit duration, min (mean $\pm \mathrm{SD})$ & $6.7 \pm 4.7$ & $6.6 \pm 5.0$ & 0.87 \\
\hline $\mathrm{HbAlc,} \mathrm{\%}$ & $8.0[7.1,9.2]$ & $9.0[7.6,9.9]$ & 0.12 \\
\hline HbAlc, $\%($ mean $\pm S D)$ & $8.0 \pm 1.1$ & $8.9 \pm 1.6$ & 0.09 \\
\hline $\mathrm{HbAlc}<8 \%$ & $7(46.7)$ & $5(33.3)$ & 0.71 \\
\hline Total daily dose increase, $\mathrm{U}^{\mathrm{b}}$ & $8.0[3.5,14.0]$ & $7.0[-0.5,12.0]$ & 0.44 \\
\hline Missed SMBG per week ${ }^{c}$ & $0.3[0.0,0.8]$ & $0.5[0.3,2.8]$ & 0.04 \\
\hline Skipped meals per week ${ }^{c}$ & $4.3[0.9,6.2]$ & $5.0[0.0,7.4]$ & 0.60 \\
\hline Mild hypoglycemia & $15(100.0)$ & $15(100.0)$ & 1.00 \\
\hline Severe hypoglycemia & $0(0.0)$ & $0(0.00)$ & - \\
\hline
\end{tabular}


Table 3 continued

\begin{tabular}{llll}
\hline Patient characteristics & Group 70 $(\boldsymbol{n}=\mathbf{1 5})$ & Group N R $(\boldsymbol{n}=\mathbf{1 5})$ & $\boldsymbol{p}$ value \\
\hline Hyperglycemic crisis $^{\mathrm{d}}$ & $0(0.0)$ & $0(0.0)$ & - \\
\hline
\end{tabular}

Values in table are shown as the median with the interquartile range in square brackets, or as a number $(n)$ with the percentage in parentheses, unless specifically stated otherwise

$B M I$ Body mass index, $H b A 1 c$ glycated hemoglobin, $S D$ Standard deviation, $S M B G$ self-monitoring blood glucose

${ }^{\text {a }}$ BMI for age $Z$-score $\geq-2$ and $<+1$

b Increase from baseline

c According to information reported on SMBG log

${ }^{\mathrm{d}}$ Includes diabetic ketoacidosis and hyperosmolar hyperglycemic state

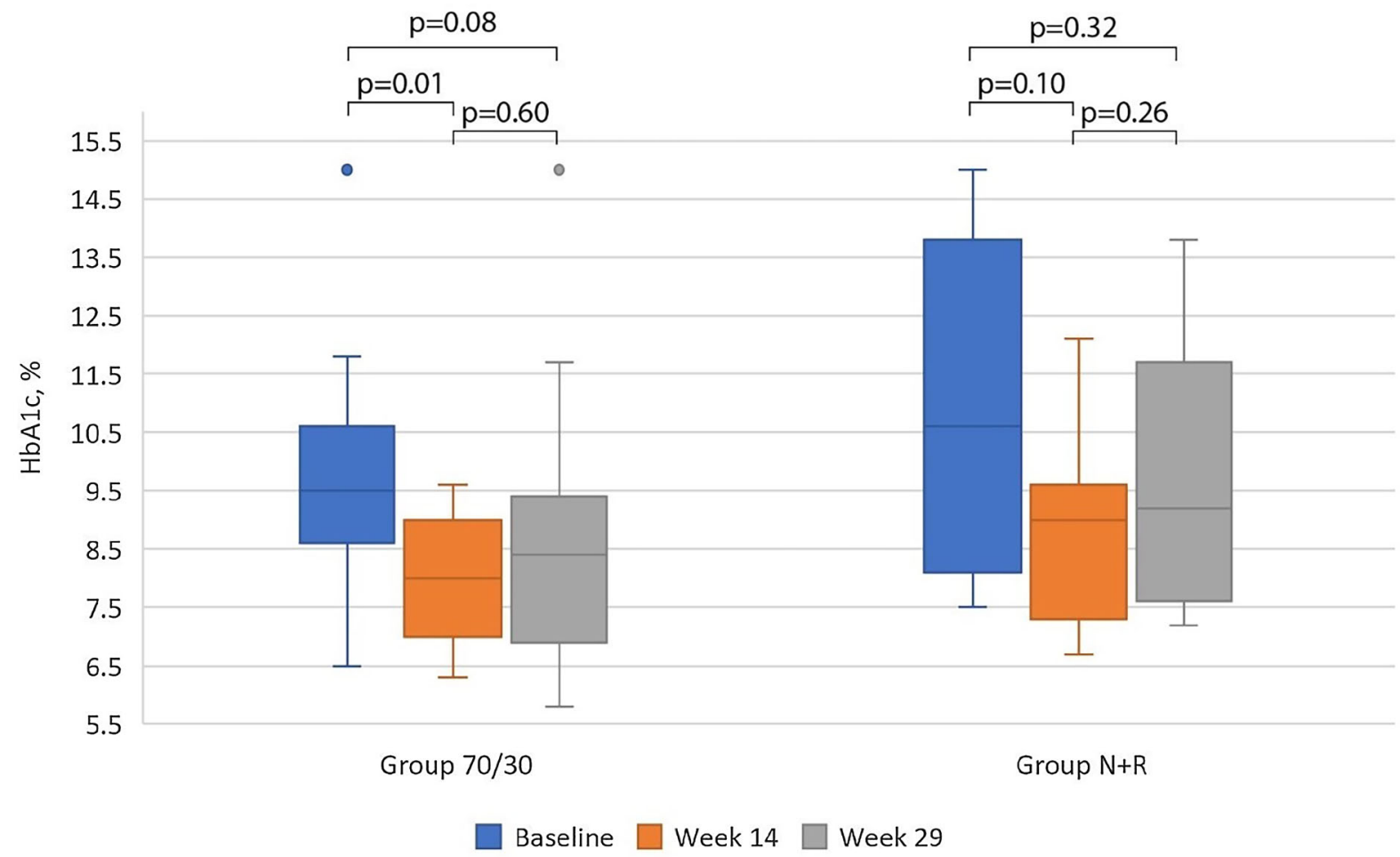

Fig. 1 Change in glycated hemoglobin (HbAlc) during the study period. In both treatment groups, HbAlc decreased significantly after 14 weeks, and re-increased to values not significantly different from baseline after 29 weeks

significant $(p=0.45)$. Large fluctuations in BG levels were not found in any patient. The median number of missed SMBG tests per week was significantly higher in GNR than G70 $(0.5$ [IQR $0.3,2.8]$ vs. 0.3 [IQR $0.0,0.8] ; p=0.04$ ). The median number of skipped meals per week did not differ between the two groups (4.3 [IQR $0.9,6.2]$ in G70 vs. 5.0 [IQR $0.0,7.4]$ in GNR; $p=$ $0.60)$. No serious acute complications, including severe hypoglycemia, were reported in either group. However, all patients reported at least one episode of mild hypoglycemia. No 
patient had to take additional insulin injections. The TDD (in units) increased in both groups from baseline, without statistical significance (G70: 8 [IQR 3.5, 14], $p=0.11$; GNR: 7 $[\mathrm{IQR}-0.5,12] ; p=0.44)$. In the multivariate regression analysis for all patients, age at evaluation, age at diagnosis, diabetes duration, $\mathrm{TDD} / \mathrm{kg}$, type of insulin regimen, BMI $Z$-score and number of missed SMBG and skipped meals did not predict $\mathrm{HbA1c}$ values.

At 29 weeks, HbA1c increased in both groups, reaching $8.4 \%$ (IQR 6.9\%, 10.2\%) (68.3 $\mathrm{mmol} / \mathrm{mol})$ and $9.2 \%$ (IQR 7.8\%, 12.0\%) $(77.0$ $\mathrm{mmol} / \mathrm{mol})$ in G70 and GNR, respectively $(p=$ 0.19) (Fig. 1). These HbA1c values were not statistically different from those at week 14 (G70: $p=0.60$; GNR: $p=0.26$ ) nor from those at baseline (G70: $p=0.08$; GNR: $p=0.32$ ).

\section{DISCUSSION}

The results of our study demonstrate that in a resource-limited setting where SMBG is infrequent, food insecurity is common and glycemic control is extremely poor, the use of a simplified sliding scale for the twice-daily administration of premixed insulin 70/30 in combination with increased frequency and duration of clinic visits significantly lowers HbA1c. The increase in HbA1c to values not significantly different from baseline at week 29 was due to the removal of the sliding scales and the return to the previous schedule of a once-monthly visit to the diabetes clinic, thereby confirming the effectiveness of the evaluated treatment protocol. The improvement in glycemic control during the intervention period was obtained without increasing the risk of hypo- or hyperglycemic events or other acute complications. However, the decrease in HbA1c with the sliding scalebased self-mixed NPH $+\mathrm{R}$ insulin regimen and the biweekly visits to the diabetes clinic did not reach statistical significance, probably because of the wide dispersion of baseline HbA1c values in the GNR, the short period of follow-up and, possibly, the persistent difficulty for some GNR patients to properly self-mix insulins, resulting in dosing errors.
While glycemic control was overall very poor and remained above the recommended target range for the vast majority of patients even during the intervention period, median HbA1c in both groups was in the expected range of mean HbA1c for an "intermediate care" environment, which is $8-9.5 \%(64-80 \mathrm{mmol} / \mathrm{mol})$ [6]. Interestingly, this result is achieved without the use of all "intermediate care" resources, which include multiple daily injections ("basalbolus regimen"). This improvement in glycemic control, if maintained, should lead to a reduction in long-term complications and mortality, as has been shown in some studies $[6,15]$. In a recent population-based cohort study, Marcus Lind found that $\mathrm{HbA} 1 \mathrm{c}>8.6 \%$ (> $70 \mathrm{mmol} /-$ mol) was a risk factor for proliferative retinopathy and macroalbuminuria in 10,398 children and adults with type 1 diabetes [16]. This finding highlights the $8 \%$ HbA1c level at week 14 in G70 in terms of reducing the risk of severe diabetic complications.

A twice-daily rather than multiple-daily insulin injection regimen was chosen for two reasons. First, glucose test strips are often unaffordable in Haiti; second, culturally, our lower-class young Haitian patients have low compliance with midday SMBG and insulin injection outside the home. They argue that the material is too cumbersome to have with them and that performing the BG test and injecting themselves with insulin injection with the knowledge of their peers is stigmatizing. A retrospective adjustment algorithm is actually among the best insulin dosing adjustment methods in terms of reducing glycemic fluctuations. However, it would be error-prone, subjective, and very difficult to apply to those receiving 70/30 insulin when meals are skipped and BG measurements missed. This is the main reason for choosing a non-typical sliding scale for this study. It is also worth noting that taking into account the insulin sensitivity factor, the $\mathrm{CHO}$ ratio and $\mathrm{CHO}$ content of the meals in the calculations establishing our sliding scale insulin regimens may have helped reduce large fluctuations in BG levels ("roller coaster" phenomenon).

Premixed human insulin regimens have been shown to be associated with a more 
important risk of hypoglycemia than self-mixed or basal-bolus regimens due to the impossibility of separately adjusting the two types of insulin to BG levels or CHO load $[11,12]$. They also provide poorer metabolic control than a selfmixed regimen when used in adolescents [4], although selection bias in these studies is possible since adolescents may have been put on premixed insulin because of non-adherence. In addition, glycemic control in teenagers is generally more difficult to achieve, likely due to a combination of social, hormonal, dietary and activity factors-even in high-income countries. For example, in the USA-based type 1 diabetes exchange registry, mean $\mathrm{HbA} 1 \mathrm{c}$ in teenagers aged $15-18$ years was $9.3 \%$ (78 $\mathrm{mmol} / \mathrm{mol})$ as compared to $8.1 \%(65 \mathrm{mmol} /-$ mol) in their 5-year-old peers [3].

Our findings suggest that in our population of youth who reside in a resource-limited, foodinsecure setting, the use of sliding scales adjusted for missed SMBG and skipped meals can be a key factor in achieving better glycemic control, without severe hypoglycemia, at least in patients treated with premixed 70/30 human insulin. The beneficial effect was achieved in the presence of a minimum but essential clinical support package that included two rather than one clinic visits per month and a moderate increase in the length of each visit for better self-management education. In resource-limited settings where children and adolescents are considered autonomous at a much younger age and patients' families have a lower education level, these findings can provide important information to diabetes care teams who are often called upon to substitute for families in terms of providing the required care support to youth with diabetes. Further, healthcare providers may encourage the transfer from a selfmixed regimen to a premixed one when the former appears to be a factor contributing to poor treatment adherence [17].

Our study has a number of limitations. The small size of the sample and the short duration of the follow-up period may have prevented us from finding significant differences between and within groups or detecting significant predictors of poor glycemic control. The high baseline HbA1c level may have made patients more prone to improving their metabolic control. Our protocol, which prevented some patients from taking insulin at midday and night in cases of missed BG tests and skipped meals, was a potential risk factor for DKA, particularly on sick days, but this risk was lessened by the systematic administration of insulin in the morning and the patients' awareness to signs and management of DKA. Similarly, failure to administer 70/30 insulin for a BG level < $80 \mathrm{mg} / \mathrm{dL}(4.4 \mathrm{mmol} / \mathrm{mol})$ in the absence of meal could result in DKA but, based on our long-standing experience at FHADIMAC, we hypothesized that rapid-acting insulin, even after ingestion of fast-acting carbohydrates, might increase the risk of hypoglycemia in the following hours. SMBG before the next meal, as recommended, then might help decrease the risk of DKA. On the other hand, the limitation of only one to two SMBG tests per day could have under-detected rates of hypoglycemia. Lastly, it was not possible to dissociate the glycemic effect of the sliding scale from that of the change in the characteristics of the visit, since the two formed a package.

\section{CONCLUSION}

The use of sliding scales adjusted for missed SMBG tests and skipped meals, and biweekly clinic visits that focus on patient self-management education significantly improved glycemic control in youth with diabetes treated with a premixed 70/30 human insulin regimen. This information may further contribute to motivating the search for new strategies to improve metabolic control in patients on premixed biphasic insulins in socioeconomic contexts where advocacy efforts to access analog insulins and greater availability of SMBG supplies remain unsuccessful.

\section{ACKNOWLEDGEMENTS}

We would like to thank the FHADIMAC's young patients for their participation in the study. 
Funding. This research did not receive any specific grant from funding agencies in the public, commercial, or not-for-profit sectors. Julia von Oettingen holds a Fonds de Recherche Sante Quebec salary award. The Rapid Service Fee was funded by the authors.

Authorship. All named authors meet the International Committee of Medical Journal Editors (ICMJE) criteria for authorship for this article, take responsibility for the integrity of the work as a whole, and have given their approval for this version to be published."

Authors' Contributions. All authors have read and approved the final manuscript. Eddy Jean-Baptiste designed the research study, participated in patient follow-up and data interpretation, wrote the first draft of the manuscript, and revised the submitted version. Philippe Larco participated in the study design, performed the statistical analysis, provided a critical review of the manuscript and revised the submitted version. Julia von Oettingen provided intellectual discussion and participated in data interpretation and manuscript writing. Graham David Ogle and Evelyne Fleury-Milfort provided intellectual discussion, participated in data interpretation and provided a critical review of the manuscript. Keddy Moïse, Rodolphe Paul, and Nancy Charles Larco participated in the study design and patient follow-up and provided a critical review of the manuscript.

Prior Presentation. Parts of the study were presented in abstract form at the 78th Scientific Sessions of the American Diabetes Association, 22-26 June 2018, Orlando, Florida (Diabetes 2018;67[Suppl 1]: https://doi.org/10.2337/ db18-93-LB).

Disclosures. Eddy Jean-Baptiste, Philippe Larco, Julia von Oettingen, Graham David Ogle, Keddy Moïse, Evelyne Fleury-Milfort, Rodolphe Paul, René Charles and Nancy Charles Larco state that they have no conflict of interest.

Compliance with Ethics Guidelines. The study protocol was reviewed and approved by the Haitian National Bioethics Committee of
Haitian Ministry of Health (Ref: 1617-55). It was performed in accordance with the Helsinki Declaration of 1964, and its later amendments. Written informed consent was obtained from adult patients and adults responsible for minors. Assent was obtained from participants younger than 18 years old.

Data Availability. All data generated or analyzed during this study are included in this published article/as supplementary information files.

Open Access. This article is licensed under a Creative Commons Attribution-NonCommercial 4.0 International License, which permits any non-commercial use, sharing, adaptation, distribution and reproduction in any medium or format, as long as you give appropriate credit to the original author(s) and the source, provide a link to the Creative Commons licence, and indicate if changes were made. The images or other third party material in this article are included in the article's Creative Commons licence, unless indicated otherwise in a credit line to the material. If material is not included in the article's Creative Commons licence and your intended use is not permitted by statutory regulation or exceeds the permitted use, you will need to obtain permission directly from the copyright holder. To view a copy of this licence, visit http://creativecommons.org/licenses/bync/4.0/.

\section{REFERENCES}

1. The Diabetes Control and Complications Trial Group. The relationship of glycemic exposure (HbA1c) to the risk of development and progression of retinopathy in the diabetes control and complications trial. Diabetes. 1995;44:968-83.

2. DiMeglio LA, Acerini CL, Codner E, et al. ISPAD Clinical Practice Consensus Guidelines 2018: glycemic control targets and glucose monitoring for children, adolescents, and young adults with diabetes. Pediatr Diabetes. 2018;19(Suppl 27):105-14.

3. Foster NC, Beck RW, Miller KM, et al. State of type 1 diabetes management and outcomes from the T1D 
exchange in 2016-2018. Diabetes Technol Ther. 2019;21:66-72.

4. Willi SM, Miller KM, DiMeglio LA, et al. Racialethnic disparities in management and outcome among children with type 1 diabetes. Pediatrics. 2015;135:424-34.

5. Borschuk AP, Everhart RS. Health disparities among youth with type 1 diabetes: a systematic review of the current literature. Fam Syst Health. 2015;33: 297-313.

6. Ogle GD, von Oettingen JE, Middlehurst AC, et al. Levels of type 1 diabetes care in children and adolescents for countries at varying resource levels. Pediatr Diabetes. 2019;20:93-8.

7. Robinson M-È, Altenor K, Carpenter C, Bonnell R, Jean-Baptiste E, von Oettingen JE. High rates of ocular complications in a cohort of Haitian children and adolescents with diabetes. Pediatr Diabetes. 2018;19:1124-30.

8. Jean-Baptiste E, Larco P, von Oettingen JE, et al. Greater age and morbidity at diagnosis of atypical diabetes compared to immune type 1 diabetes in Haitian youth. In: International Diabetes Federation Congress, 4-8 December 2017, Abu Dhabi

9. Life For A Child (L.F.A.C.). https://www. lfacinternational.org/. 2019. Accessed 01 Aug 2019.

10. Wolfsdorf JI, Allgrove J, Craig ME, et al. ISPAD Clinical Practice Consensus Guidelines 2014: diabetic ketoacidosis and hyperglycemic hyperosmolar state. Pediatr Diabetes. 2014;15(Suppl 20):154-79.
11. Ly TT, Maahs DM, Rewers A, Dunger D, Oduwole A, Jones TW. ISPAD Clinical Practice Consensus Guidelines 2014: assessment and management of hypoglycemia in children and adolescents with diabetes. Pediatr Diabetes. 2014;15(Suppl 20): 180-92.

12. Davidson PC, Hebblewhite HR, Steed RD, Bode BW. Analysis of guidelines for basal-bolus insulin dosing: basal insulin, correction factor, and carbohydrate-to-insulin ratio. Endocr Pract. 2008;14: 1095-101.

13. Walsh J, Roberts R, Varma C, Bailey T. Using insulin. San Diego: Torrey Pines Press; 2003.

14. Walsh J, Roberts R. Pumping insulin. San Diego: Torrey Pines Press; 1994.

15. Gregory GA, Guo J, Klatman EL, et al. Costs and outcomes of "intermediate" vs "minimal" care for youth-onset type 1 diabetes in six countries. Pediatr Diabetes. 2020;21:628-36.

16. Lind M, Pivodic A, Svensson A, Ólafsdóttir AF, Wedel $\mathrm{H}$, Ludvigsson $\mathrm{J} \mathrm{HbA}_{1 \mathrm{c}}$ level as a risk factor for retinopathy and nephropathy in children and adults with type 1 diabetes: Swedish populationbased cohort study. BMJ. 2019. https://doi.org/10. 1136/bmj.14894.

17. Turner HE, Matthews DR. The use of fixed-mixture insulins in clinical practice. Eur J Clin Pharmacol. 2000;56:19-25. 